\title{
Chromothripsis and progression-free survival in metastatic colorectal cancer
}

\author{
ELINA SKUJA $^{1,2}$, DAGNIJA KALNIETE ${ }^{2}$, MIKI NAKAZAWA-MIKLASEVICA ${ }^{2}$, \\ ZANDA DANEBERGA $^{2}$, ARNIS ABOLINS ${ }^{3}$, GUNTA PURKALNE ${ }^{1,2}$ and EDVINS MIKLASEVICS ${ }^{2}$ \\ ${ }^{1}$ Clinic of Oncology, Pauls Stradins Clinical University Hospital, LV-1002 Riga; ${ }^{2}$ Institute of Oncology, Riga Stradins University, \\ LV-1007 Riga; ${ }^{3}$ Institute of Pathology, Pauls Stradins Clinical University Hospital, LV-1002 Riga, Latvia
}

Received August 29, 2016; Accepted October 26, 2016

DOI: $10.3892 / \mathrm{mco} .2017 .1123$

\begin{abstract}
Metastatic dissemination of the primary tumor is the major cause of death in colorectal cancer (CRC) patients. Multiple chromosomal breaks and chromothripsis, a phenomenon involving multiple chromosomal fragmentations occurring in a single catastrophic event, are associated with cancer genesis, progression and developing of metastases. The aim of this study was to evaluate the effect of chromothripsis and total breakpoint count (breakpoint instability index) on progression-free survival (PFS). A total of 19 patients with metastatic CRC (mCRC) receiving FOLFOX first-line palliative chemotherapy between August, 2011 and October, 2012 were selected for this study. The results indicated that the highest breakpoint count was observed in chromosomes 1, 2 and 6. Chromothripsis was detected in $52.6 \%$ of the study patients. Furthermore, chromothripsis was associated with an increased median PFS (mPFS; 14 vs. 8 months, respectively; $\mathrm{P}=0.03$ ), but an association with overall survival was not identified. The present study demonstrated that chromothripsis affected CRC patient survival, suggesting a role for this event as a prognostic and predictive marker in mCRC treatment.
\end{abstract}

\section{Introduction}

Mortality from colorectal cancer (CRC) has decreased over the past 30 years; however, there is a significant heterogeneity in survival rates that may be mainly explained by variations in patient and tumor characteristics, host response factors and applied treatment modalities. At the time of diagnosis, $\sim 25 \%$ of the patients have already developed metastases and $\sim 50 \%$ will develop metastases following treatment of the primary CRC (1).

Correspondence to: Dr Elina Skuja, Clinic of Oncology, Pauls Stradins Clinical University Hospital, 13 Pilsonu Street, LV-1002 Riga, Latvia

E-mail: elinaskuja@inbox.lv

Key words: metastatic colorectal cancer, chromothripsis, breakpoint instability index, progression-free survival
During cancer development, the cells acquire multiple point mutations and chromosomal rearrangements. Chromosomal abnormalities that are found in primary CRC cells are gains of $8 \mathrm{q}, 11 \mathrm{q}, 13 \mathrm{q}$ and $20 \mathrm{q}$ and losses of $1 \mathrm{p}, 4,8 \mathrm{p}, 17 \mathrm{p}, 18 \mathrm{q}$ and $22 \mathrm{q}$ chromosomal regions (2-5).

Van den Broek et al reported a clinical relevance of chromosomal breaks in metastatic CRC (mCRC) in 2015; they identified 1605 genomic breakpoint locations and 748 breakpoint genes with recurrence in multiple CRC samples. None of the individual breakpoint genes was significantly associated with overall survival (OS), but four CRC subtypes were revealed based on recurrent breakpoint genes and mutation status of commonly affected CRC genes [adenomatous polyposis coli, tumor protein $\mathrm{p} 53$, Kirsten rat sarcoma viral oncogene homolog (KRAS), phosphatidylinositol-4,5-bisphosphate 3 -kinase catalytic subunit $\alpha$, B-Raf proto-oncogene, serine/threonine kinase, neuroblastoma RAS viral oncogene homolog, mothers against decapentaplegic homolog 4 and F-box/WD repeat-containing protein 7] (6).

Chromothripsis, a massive chromosome fragmentation occurring in one catastrophic event, is observed in 2-3\% of cancers (7). Such genomic rearrangements may drive the development of cancer through several mechanisms, including deletion of tumor suppressor genes and increased copy number of oncogenes. The prevalence of chromothripsis and its effect on prognosis and metastasis is unclear. The incidence of chromothripsis ranges from $1.3 \%$ in multiple myeloma (8) to $33 \%$ in osteosarcoma (7). Furthermore, chromothripsis has been associated with poor patient survival in the aggressive triple-negative subtype of breast cancer, multiple myeloma and pediatric medulloblastoma (8-10), indicating its potential relevance as a prognostic marker, and suggesting chromothripsis to be a characteristic of certain particularly aggressive types of cancer.

The incidence of chromothripsis and its effect on survival in $\mathrm{CRC}$ is unclear, but it may be a prevalent genomic rearrangement in this type of cancer (11).

\section{Patients and methods}

Study population. A total of $19 \mathrm{mCRC}$ patients who received chemotherapy at the Clinic of Oncology of Pauls Stradins Clinical University Hospital (Riga, Latvia) between August, 2011 and October, 2012 were selected. The study was 
Table I. Clinical and biological characteristics of the patients $(n=19)$.

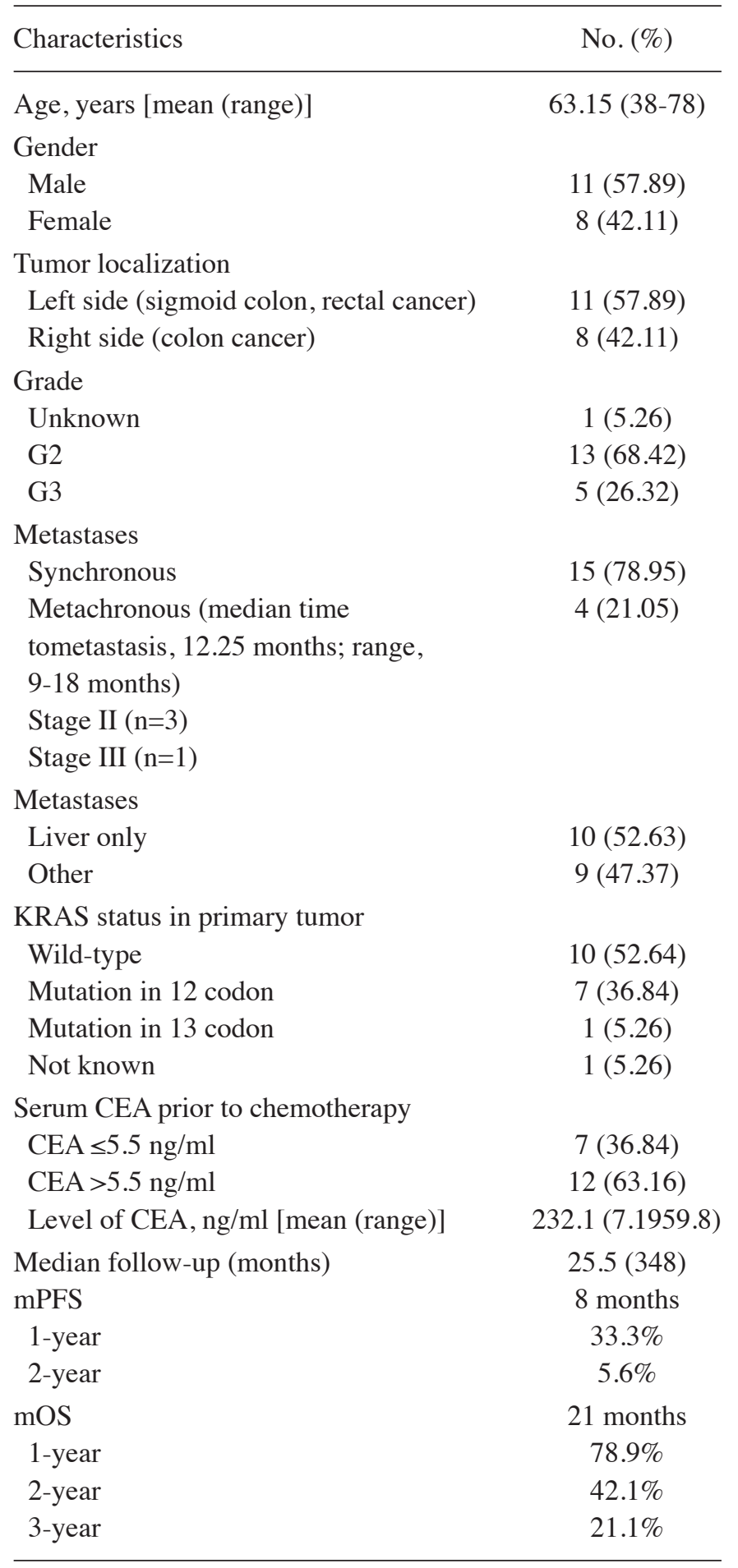

CEA, carcinoembryonic antigen; KRAS, Kirsten rat sarcoma viral oncogene homolog; mPFS, median progression-free survival; mOS, median overall survival.

performed with approval from the Ethics Committee of the Riga Stradins University and written informed consent was obtained from all the patients who were investigated. Tissue samples were acquired as part of a series of routine diagnostic and pathological analyses at the hospital. The patients were followed up for 3-48 months.
Table II. Chromosomes affected by chromothripsis and total breakpoint count per chromosome.

\begin{tabular}{rcc}
\hline Chromosome & $\begin{array}{c}\text { Breakpoint no., } \\
\text { range (median) }\end{array}$ & $\begin{array}{c}\text { Chromothripsis, } \\
\text { no. of patients }(\%)\end{array}$ \\
\hline 1 & $27-365(150.2)$ & $10(52.6)$ \\
2 & $25-315(136.8)$ & $10(52.6)$ \\
3 & $21-234(94.5)$ & $7(36.8)$ \\
4 & $10-232(73.9)$ & $5(26.3)$ \\
5 & $20-189(87.1)$ & $5(26.3)$ \\
6 & $22-298(118.9)$ & $10(52.6)$ \\
7 & $16-195(82.7)$ & $5(26.3)$ \\
8 & $16-199(81.6)$ & $5(26.3)$ \\
9 & $11-205(79.2)$ & $5(26.3)$ \\
10 & $21-275(106.8)$ & $7(36.8)$ \\
11 & $34-252(104.1)$ & $8(42.1)$ \\
12 & $18-206(84.2)$ & $6(31.6)$ \\
13 & $14-166(54.1)$ & $3(15.8)$ \\
14 & $12-152(58.6)$ & $4(21.1)$ \\
15 & $3-166(58.9)$ & $4(21.1)$ \\
16 & $9-158(60.5)$ & $4(21.1)$ \\
17 & $10-206(66.8)$ & $4(21.1)$ \\
18 & $10-149(50.5)$ & $2(10.5)$ \\
19 & $7-137(44.2)$ & $2(10.5)$ \\
20 & $9-124(42.3)$ & $2(10.5)$ \\
21 & $7-99(24.7)$ & $0(0.0)$ \\
22 & $4-131(37)$ & $1(5.3)$ \\
\hline & & \\
\hline & & \\
\hline
\end{tabular}

The clinical and biological characteristics of the patients are summarized in Table I. Of the 19 patients, 15 had primary metastatic cancer (stage IV), whereas the 4 remaining patients developed metastases after treatment of the primary cancer. A total of 10 patients developed metastases only to the liver; 16 patients underwent primary tumor surgery, whereas in 3 patients biopsy alone was performed. In 7 patients, the carcinoembryonic antigen (CEA) level was $\leq 5.5 \mathrm{ng} / \mathrm{ml}$ prior to chemotherapy.

A total of 18 patients received FOLFOX first-line chemotherapy (1 patient declined chemotherapy). After disease progression, 13 patients received irinotecan-containing second-line chemotherapy, 1 patient was rechallenged with FOLFOX, 1 patient received oral fluoropyrimidine therapy with Ftorafur (tegafur) and 1 patient received best supportive care. Only 5 patients $(26.3 \%)$ received third-line therapy [irinotecan, oxaliplatin or 5-fluorouracil (5FU)]. One patient underwent hepatic surgery for CRC metastases after discontinuation of second-line chemotherapy, 2 patients received salvage transcatheter arterial chemoembolization of CRC liver metastases by irinotecan-eluting microspheres, and 1 patient received palliative radiotherapy for local rectal cancer. Data on clinical follow-up were obtained until August, 2016.

Genotyping. DNA was extracted from formalin-fixed paraffin-embedded (FFPE) samples with QIAamp DNA Mini kit (Qiagen, Hilden, Germany) according to the 

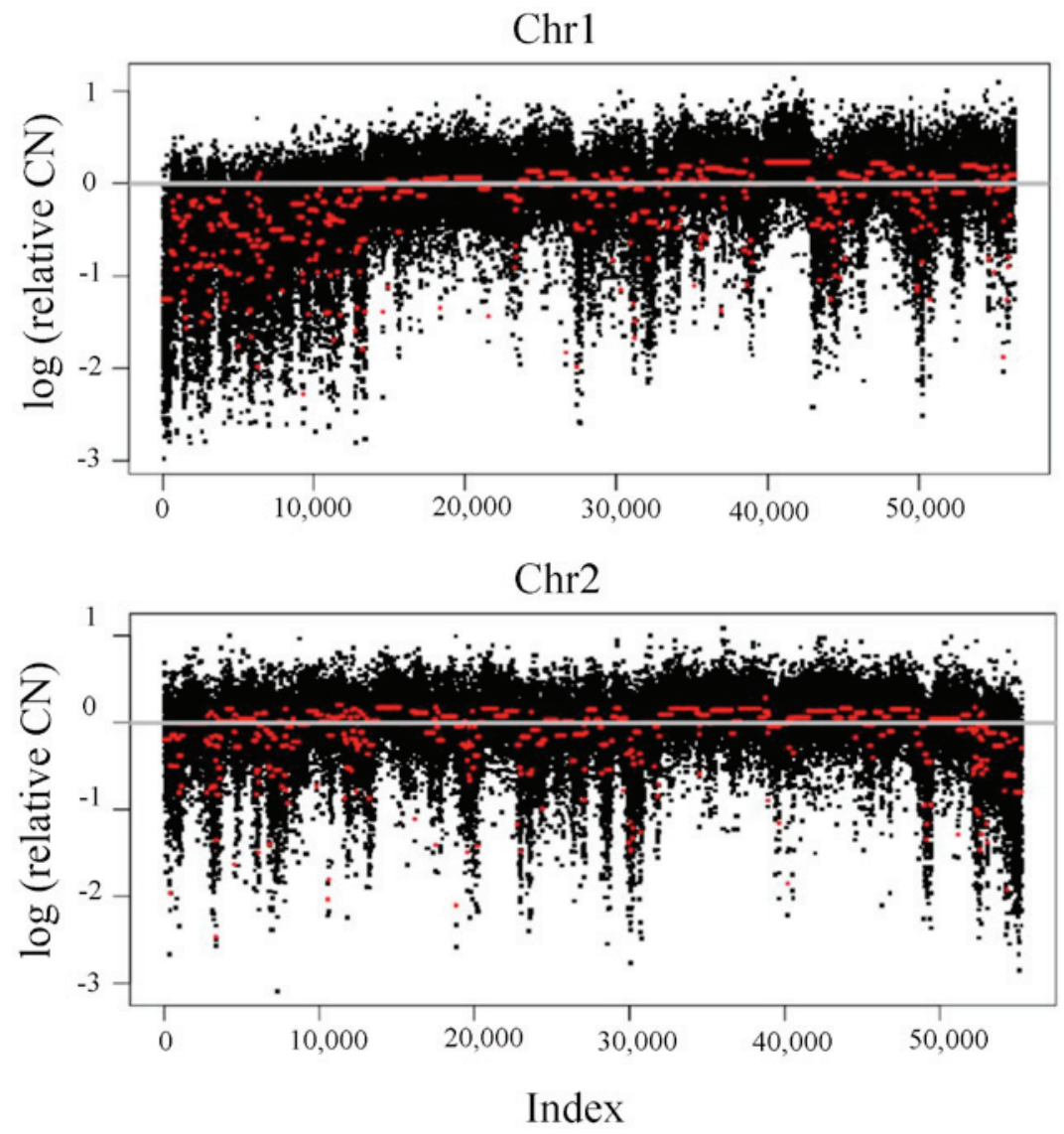

Figure 1. Multiple chromosome 1 fragmentation (chromothripsis). There were multiple deletions in both arms of chromosomes 1 and 2 in patient 14 . Chr, chromosome; $\mathrm{CN}$, copy number.
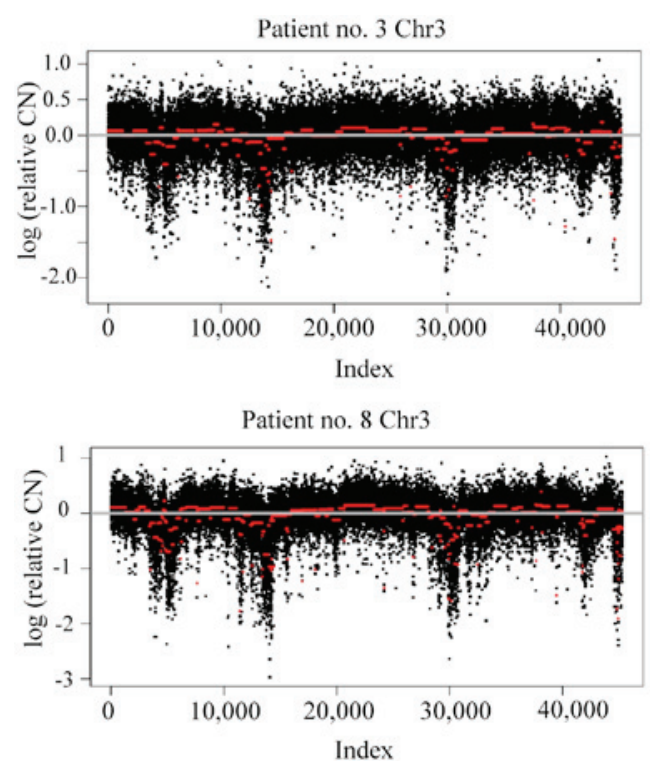
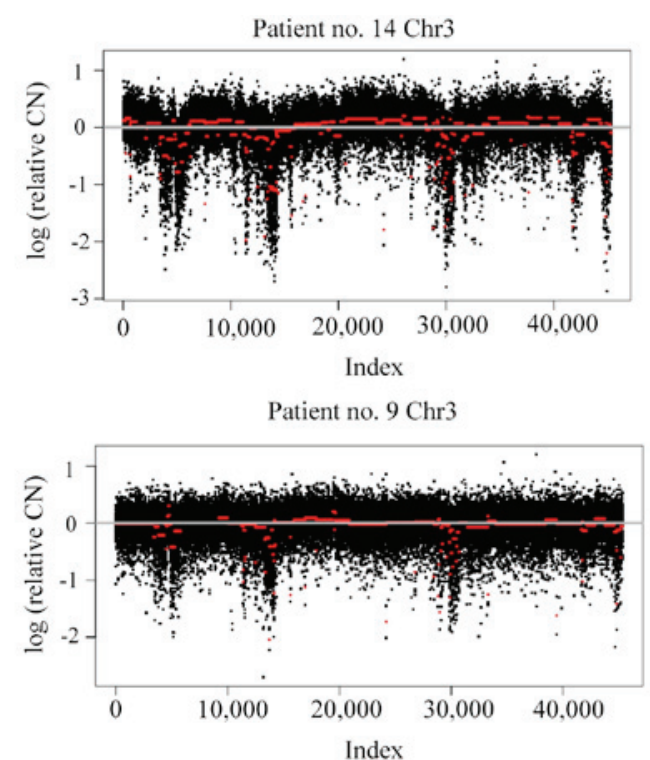

Figure 2. Similar pattern of chromosomal rearrangements of chromosome 3 in four different patients (patients 3, 8, 9 and 14). Chr, chromosome; CN, copy number.

manufacturer's instructions. Quality was evaluated using the Illumina FFPE QC kit (Illumina, San Diego, CA, USA) by reverse transcription-polymerase chain reaction. DNA was restored with the Illumina DNA restoration kit (Illumina). Microarray analysis was performed using the Infinium
HumanOmniExpress-12 v1.0 FFPE BeadChip kit (Illumina). BeadChip was scaned on HiScan (Illumina). Analysis was performed by GenomeStudio software (Illumina) and $\mathrm{R}$ version 3.1.2. (https://www.r-project.org/). Copy number variation and breakpoints on the chromosomes were analyzed 
A

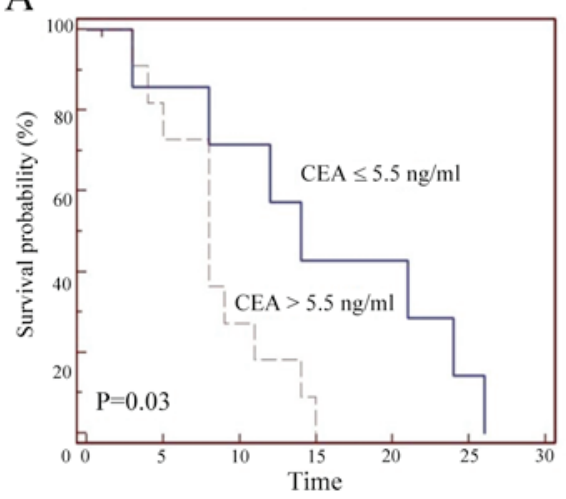

B

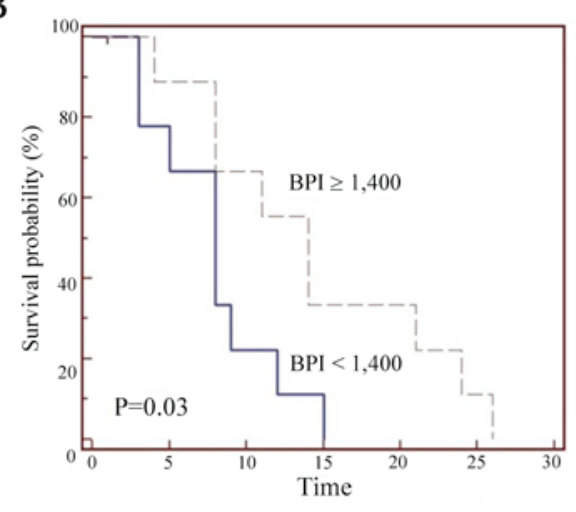

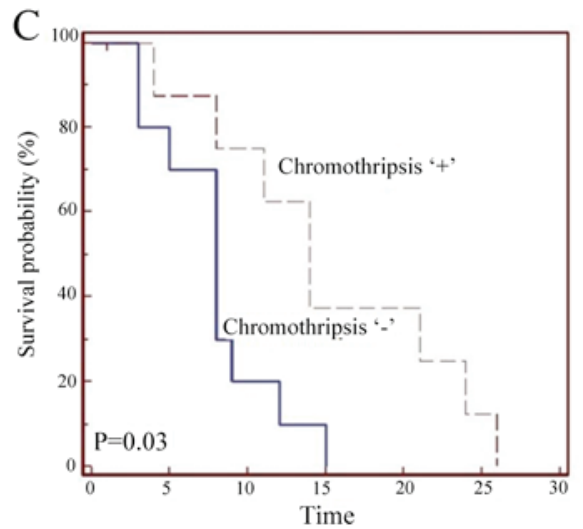

Figure 3. (A) mPFS in elevated CEA: 8 months; mPFS in CEA $\leq 5.5 \mathrm{ng} / \mathrm{ml}: 14$ months; HR=0.29. (B) mPFS for BPI $\geq 1,400: 14$ months; mPFS for BPI <1,400: 8 months; HR=3.43. (C) mPFS in cases exhibiting chromothripsis (+): 14 months; mPFS in cases without chromothripsis (-): 8 months; HR=3.43. mPFS, median progression-free survival; CEA, carcinoembryonic antigen; BPI, breakpoint instability index; HR, hazard ratio.

using the DNA copy package (http://bioconductor.org/packages/release/bioc/html/DNAcopy.html).

OS and progression-free survival (PFS) rates were estimated using the Kaplan-Meier method. The log-rank test was used to calculate any significant difference between the subgroups by univariate analysis. Significance levels were set at $\mathrm{P}<0.05$. All statistical analyses were performed using MedCalc software, version 16.4.8 (MedCalc Software, Ostend, Belgium).

\section{Results}

Breakpoint count per chromosome. The aim of this study was to evaluate the total number of chromosomal breaks and association of chromothripsis ( $>100$ breakpoints detected in one chromosome) with PFS in mCRC. The total number of breakpoints per genome in cancer tissue [breakpoint instability index (BPI)] was 368-4,009. The highest breakpoint count was seen in chromosome 1 (27-365 breakpoints), followed by chromosome 2 (25-315) (Table II), indicating the crucial role of these chromosomes in CRC development. The lowest density of breaks occurred in chromosome 21 (7-99 breakpoints; Fig. 1). Of note, a similar pattern of chromosomal rearrangements was observed in several patients for chromosomes 1, 3 and 8 (Fig. 2).

In 10 of the tumor samples (52.6\%), multiple chromosomal fragmentations were found, found, referred to as chromothripsis, a recently reported catastrophic genetic event. The most commonly affected chromosomes were chromosomes 1 , 2 and 6 (52.6\% of the patients; Table II). The maximal count of chromosomes affected by chromothripsis was 20 , which was observed in 1 patient.

Association of breakpoint number with clinicopathological characteristics. No association of BPI value and chromothripsis with cancer localization (rectal or colon cancer), CEA level, KRAS mutational status in primary cancer and stage [synchronous metastatic disease (stage IV) vs. metachronous metastatic disease] was observed.

Association of breakpoint number with survival. PFS and OS were measured for all the patients in study. Decreased PFS was observed in patients with left-sided metastatic cancer (sigmoid colon and rectal cancer), elevated CEA level prior to chemotherapy (Fig. 3A) and KRAS mutations.

A positive correlation between high BPI (chromothripsis) and increased PFS was observed (Fig. 3B and C). Due to the observed better PFS in different clinical subgroups by CEA, KRAS status and primary tumor location, the effect of BPI and chromothripsis on clinical findings was analyzed; however, the number of patients in each subgroup was insufficient to establish a statistically significant correlation. In patients with detected chromothripsis and CEA $\leq 5.5 \mathrm{ng} / \mathrm{ml}$ $(n=4)$, a median PFS (mPFS) of 22.5 months was observed; in patients without chromothripsis and elevated CEA level $(n=7)$, an mPFS of 8 months was observed, but these findings did not reach statistical significance. A statistically significant effect of any clinical or biological factors on OS was not observed.

\section{Discussion}

The effect of chromosomal rearrangements and mutations on pathogenesis, prognosis and resistance to treatment are widely described in mCRC studies. The prognostic and predictive role of breakpoint instability index and chromothripsis remains unclear.

In the present study, a correlation between massive DNA fragmentation (chromothripsis) and PFS in mCRC was observed. As opposed to recent studies suggesting chromothripsis to be associated with worse prognosis, we found chromothripsis to be a positive predictive factor for first-line chemotherapy. It may be hypothesized that cancer cells exhibiting radical DNA rearrangements, such as chromothripsis, are more sensitive to nucleic acid-damaging therapy with 5FU and oxaliplatin.

Breakpoint instability index was previously reported in breast cancer study by Przybytkowski et al (10); they reported a BPI of 25-300 in breast cancer tissue, with the highest density of breaks in chromosome 17 and the lowest density in chromosome 4. The BPI appeared to be different in different breast cancer molecular subtypes, with the highest breakpoint count in aggressive triple-negative breast cancer. In comparison, in our study on CRC tissue, the highest breakpoint density was found in chromosomes 1 and 2 and the lowest in chromosome 21 , but the BPI was significantly higher (368-4,009). In 
addition, 10 tumor samples (52.6\%) exhibited a chromothripsis pattern on $\geq 3$ chromosomes, which was higher compared with previous reports $(7,8,10)$. The high BPI value and high prevalence of chromothripsis in our study may be attributed to the fact that all the patients had late-stage metastatic disease, which is consistent with the prevalence of chromothripsis reported in high-risk aggressive tumors.

In conclusion, the present study demonstrated that chromothripsis is associated with increased PFS, but not with OS in mCRC. Further studies with larger sample sizes are required to determine whether chromothripsis and BPI value may be used as a prognostic or predictive factor for oxaliplatin-containing first-line chemotherapy for $\mathrm{mCRC}$.

\section{Acknowledgements}

The present study was partly supported by the National Research Program 'Biomedicine for Public Health (BIOMEDICINE)'.

\section{References}

1. Khatri VP, Petrelli NJ and Belghiti J: Extending the frontiers of surgical therapy for hepatic colorectal metastases: Is there a limit? J Clin Oncol 23: 8490-8497, 2005.

2. Sayagués JM,Fontanillo C, Abad Mdel M, González-González M, Sarasquete ME, Chillon MdC, Garcia E, Bengoechea O, Fonseca E, Gonzalez-Diaz M, et al: Mapping of genetic abnormalities of primary tumours from metastatic CRC by high-resolution SNP arrays. PLoS One 5: e13752, 2010.

3. Sylvester BE and Vakiani E: Tumor evolution and intratumor heterogeneity in colorectal carcinoma: Insights from comparative genomic profiling of primary tumors and matched metastases. J Gastrointest Oncol 6: 668-675, 2015.
4. González-González M, Muñoz-Bellvis L, Mackintosh C, Fontanillo C, Gutiérrez ML, Abad MM, Bengoechea O, Teodosio C, Fonseca E, Fuentes M, et al: Prognostic impact of del (17p) and del (22q) as assessed by interphase FISH in sporadic colorectal carcinomas. PLoS One 7: e42683, 2012.

5. Ali Hassan NZ, Mokhtar NM, Kok Sin T, Mohamed Rose I, Sagap I, Harun R and Jamal R: Integrated analysis of copy number variation and genome-wide expression profiling in colorectal cancer tissues. PLoS One 9: e92553, 2014.

6. van den Broek E, Dijkstra MJ, Krijgsman O, Sie D, Haan JC, Traets JJ, van de Wiel MA, Nagtegaal ID, Punt CJ, Carvalho B, et al: High prevalence and clinical relevance of genes affected by chromosomal breaks in colorectal cancer. PLoS One 10: e0138141, 2015.

7. Stephens PJ, Greenman CD, Fu B, Yang F, Bignell GR, Mudie LJ, Pleasance ED, Lau KW, Beare D, Stebbings LA, et al: Massive genomic rearrangement acquired in a single catastrophic event during cancer development. Cell 144: 27-40, 2011.

8. Magrangeas F, Avet-Loiseau H, Munshi NC and Minvielle S: Chromothripsis identifies a rare and aggressive entity among newly diagnosed multiple myeloma patients. Blood 118: 675-678, 2011.

9. Rausch T, Jones DT, Zapatka M, Stütz AM, Zichner T, Weischenfeldt J, Jäger N, Remke M, Shih D, Northcott PA, et al: Genome sequencing of pediatric medulloblastoma links catastrophic DNA rearrangements with TP53 mutations. Cell 148: 59-71, 2012.

10. Przybytkowski E, Lenkiewicz E, Barrett MT, Klein K, Nabavi S, Greenwood CM and Basik M: Chromosome-breakage genomic instability and chromothripsis in breast cancer. BMC Genomics 15: 579, 2014

11. Kloosterman WP, Hoogstraat M, Paling O, Tavakoli-Yaraki M, Renkens I, Vermaat JS, van Roosmalen MJ, van Lieshout S, Nijman IJ, Roessingh W, et al: Chromothripsis is a common mechanism driving genomic rearrangements in primary and metastatic colorectal cancer. Genome Biol 12: R103, 2011. 